Tanzania Journal of Science 47(3): 943-952, 2021
ISSN 0856-1761, e-ISSN 2507-7961

\title{
Efficient Load Balancing Algorithm in Long Term Evolution (LTE) Heterogeneous Network Based on Dynamic Cell Range Expansion Bias
}

\author{
Emmanuel Bakuza*, Hashimu U. Iddi and Abdi T. Abdalla \\ Department of Electronics and Telecommunications Engineering, College of Information and \\ Communication Technologies, University of Dar es Salaam, Tanzania \\ E-mail addresses: emmanuelbakuza@gmail.com, hashimuledi@udsm.ac.tz,abdit@udsm.ac.tz \\ *Corresponding author \\ Received 29 Mar 2021, Revised 12 Jul 2021, Accepted 20 Jul 2021, Published Aug 2021 \\ DOI: https://dx.doi.org/10.4314/tjs.v47i3.6
}

\begin{abstract}
The traditional scheme for load balancing in a homogeneous Long Term Evolution (LTE) Network where User Equipment (UEs) associate to a node with the strongest received signal strength is not practical for LTE Heterogeneous Network (LTE HetNet) due to power disparity between the nodes. Therefore, dynamic Cell Range Expansion (CRE) based load-balancing schemes were employed by several scholars to address the challenges in the LTE HetNet. However, the fairness index in achieving the desired average user throughput and UE offloading effect is relatively low. In this work, an efficient load-balancing algorithm for LTE HetNet based on dynamic Cell Range Expansion (CRE) was developed to improve the fairness of the network for the desired throughput and UE offloading effect. The simulation results achieve a throughput gain improvement of up to $11 \%$, while the fairness index improves by $6 \%$ compared to the existing algorithm. Further, the UEs offloading effect shows a significant improvement of 3\% relative to the existing algorithm.
\end{abstract}

Keywords: Fairness Index, Cell Range Expansion, Load Balancing, LTE Heterogeneous Network, Throughput.

\section{Introduction}

The ramp up of data traffic demands in recent years calls for innovative system designs for more capacity in wireless cellular networks (Klaine et al. 2017). In response to these requirements, 3GPP introduced Long Term Evolution (LTE) advanced standard, which is built on old LTE standards (Hasiandra and Iskandar 2017). To meet the requirements for capacity enhancement in LTE, it went through major technology upgrades in the radio interface (Ramaswamy and Correia 2019). As one of the upgrades for capacity enhancements, included heterogeneous networks (HetNets) in the radio access network of LTE (Bendaoud et al. 2018). In HetNets High Power Nodes (HPNs) and Low Power Nodes (LPNs) are deployed in the same location, where typically HPNs cells provide an umbrella coverage for a large area, overlaid with LPNs provide smaller cells to enhance network capacity or extend network coverage (Padaganur and Mallapur 2017).

Due to uneven load distributions in LTE HetNet, load balancing is key to equitable sharing of load amongst deployed nodes of a radio access network (Alam et al. 2018). Load balancing is the process of equitably distributing load in a network to ensure that resources are efficiently utilized (Kaur and Sharma 2018). The power disparity between the nodes in LTE HetNet has caused the traditional load balance scheme strategy, whereby UEs associate to a node with the 
strongest received signal strength to be impractical (Lai and Liu 2018). This is because, it creates load imbalance between these nodes which impact the performance of LTE HetNet in terms of fairness index value, user throughput rate and UE offloading effect (Hammad et al. 2018).

Therefore, several approaches on load balance scheme based on Cell Range Expansion (CRE) were employed to address the challenge. The authors $\mathrm{Ni}$ et al. (2018) proposed a novel downlink cell-edge-aware zero forcing (CEA-ZF) and Block Diagonalization (BD) cooperative precoding scheme to suppress the downlink interference caused by small cell range expansion and maximize the overall system UE sum-rate. However, simulation results highlighted degradation of network fairness value as compared to the existing approaches.

Afterward, Huang and Zhang (2019) proposed CRE approach based on dual connectivity (DC) in two-tier LTE HetNet to enhance the SINR of the biased users, and the simulation results in terms of coverage probability, average user throughput and area spectral efficiency outperform the benchmarked scheme, however, fairness index values were degraded.

In Kuribayashi et al. (2020), the authors coupled CRE technique along with a Particle Swarm Optimization (PSO) algorithm to maximize the number of users whose downlink requirements are met. The proposed algorithm is also compared with some classical PSO implementations. Simulation results validate the performance of the proposed algorithm, which effectively fulfills user throughput performance at the cost of network fairness values.

In this work, an efficient load balancing algorithm based on dynamic CRE bias has been developed to improve the fairness of the LTE HetNet for the desired user throughput rate and UE offloading effect. An automatic dynamic bias value was calculated which dynamically balances UEs as per the current load status of the network using an adjustment function.

\section{Materials and Methods System model}

The system is modelled such that LPNs and HPNs are deployed on the same channel and their interference coordination is not considered, as the focus of this work is to investigate load balancing by user association. Interference coordination, however, will be the future extension.

Both nodes assumed to be connected to the core network, that is Evolved Packet Core (EPC) via a backhaul (S1 interface) which may be a physical or wireless link and can communicate amongst them via the $\mathrm{X} 2$ interface. The purpose of the X2 interface is to communicate load status information and transmit data during UE association, as shown in Figure 1. Also, the Mobility Management Entity (MME) performs Non-Access Stratum (NAS) signaling between the UE and EPC. The Serving Gateway (S-GW) performs routing and forwarding of packets coming to and leaving the UE.

For UEs to associate with suitable nodes, cell selection or handover occurs. In LTE, handover is only performed if UE is currently in dedicated connected mode; otherwise, cell selection is performed (Netalkar et al. 2020). A handover procedure between cells that share the same MME and have an X2 interface is normally implemented via the $\mathrm{X} 2$ interface (Mannem et al. 2020). However, if the X2 interface is not available, the S1-C interface is used (Kosmopoulos et al. 2020). The basic stages of an LTE X2 handover procedure are divided into four. In the first stage, UE measures the Reference Signal Received Power (RSRP) from the downlink channel and reports to the node (Alhammadi et al. 2020). Then in the second stage, the source node prepares for a handover to a target node and forwards user and control plane contexts. In the third stage, the source node sends a handover command to the UE (Pal et al. 2020). During the period when the UE processes the handover command and the time when the target node confirms the handover, there is a brief break of UE 
connectivity. Then finally, it connects to the target cell and the session is restored (Dogra et al. 2020).

In the physical layer, an Orthogonal Frequency-Division Multiplexing (OFDMA) system is considered, where all the nodes are assumed to have the same bandwidth. The bandwidth is split into smaller sub-carriers, which are grouped into 12 units and scheduled as resource blocks. Physical Resource Blocks (PRB) are the basic time-frequency resource units that can be assigned to UEs. Conventionally, UEs associate with the nodes that have the highest measured RSRP value.

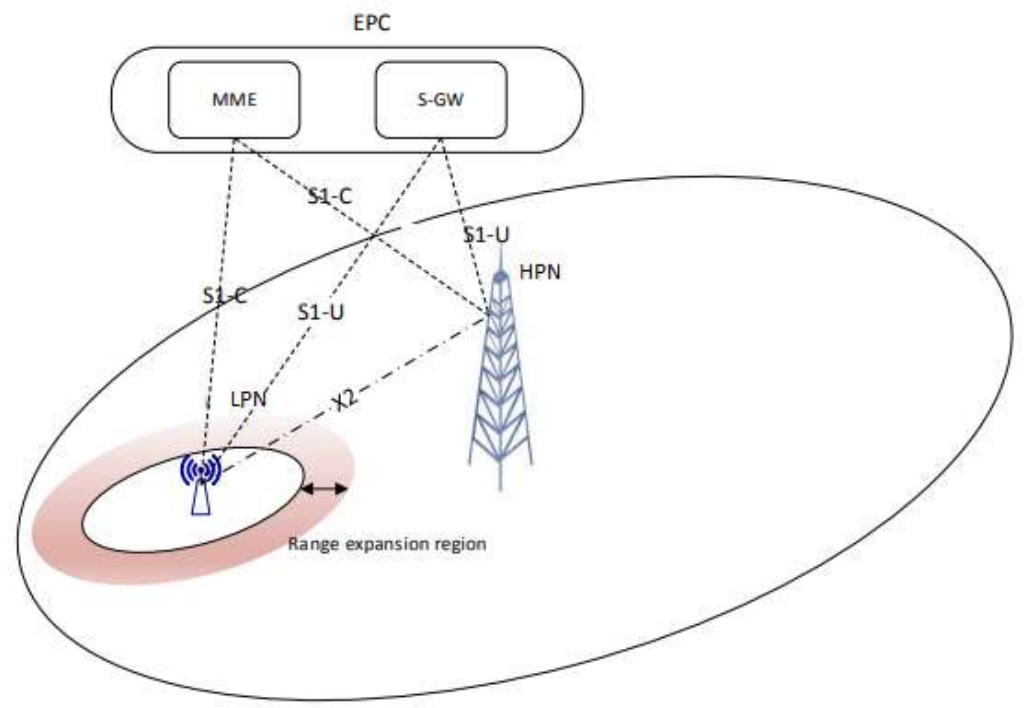

Figure 1: CRE bias scenario in Vienna LTE System Level simulator (Ramaswamy and Correia 2019).

In LTE HetNets, Markov processes have been used in modelling handover. With extension, Markov processes can be used in load balancing problems. The fundamental understanding of the load status concept to varying Cell Range Bias for load balancing can be deduced such that a UE associates with a node that maximizes its utility. The association rule $U E_{i j}$, to the nodes $\mathrm{i}$ and $\mathrm{j}$ can be defined in Equation (1) (Bhuvaneswari et al. 2015);

$$
U E_{i j}=\arg \max _{U}\left(U_{i j}-\lambda_{j}\right)
$$

where $U_{i j}$ defines the utility of the UE that attached to the nodes $i$ and $j$, and $\lambda_{j}$ represents the traffic density of the LPNs. It is clear, to this point, that the traffic density influences the association decisions of the UEs (Thienthong et al. 2019). Least Mean Square (LMS) approach was used to design an adaptive scheduler for the systems. The basic idea behind the LMS approach is to determine the optimal utility (Abohamra et al. 2017). This is achieved by updating the utility in such a manner that the system converges to an optimal solution. We use this expression to obtain the utility in our algorithm. According to the rule, each UE chooses a node that offers the highest utility less than the traffic density for all the possible user-cell associations. Therefore, the low traffic density tends to increase the utility, thereby incentivizing UEs to associate with LPNs, while high traffic density does the opposite. Based on curve fitting with Matlab, the utility varies exponentially with traffic density. Therefore, on the expansion of the utility $U_{i j}$, the expression in Equation (1) can alternatively be written as Equation (2). 


$$
U E_{i j}=\arg \max _{U}\left(U_{i j} e^{-\lambda_{j}}\right)
$$

The aim is to achieve realistic UE-cell associations that provide sufficient load balancing at the cost of minimal association errors. Therefore, the association rule of UE to LPN is now defined as (3)

$$
\begin{aligned}
& \text { Cell }_{\text {dynamic }}= \\
& \arg \max _{R S R P}\left\{R S R P_{j}+\text { Bias }_{j}\right\}
\end{aligned}
$$

where Bias $_{j}=\beta+\beta\left(e^{-\lambda_{j}}\right)$ and $\beta$ is positive coefficient that controls the extent to which the bias could be aggressive.

For the traffic offloading impact that CRE has on multi-tiered networks, we set all HPN biases to zero, and the algorithm is predominantly executed in LPN. The role of the HPN is to ensure synchronization of load balancing related processes in LPNs. A high value of $\beta$, for instance, will result in an aggressive user-cell association. In contrary, a very low value of $\beta$ will result in a sluggish up-take of users during the UE-cell association process. Aggressive UE-cell association is not always desirable, especially in the cell edge of range-expanded regions of LPNs. Since the nodes experience spatial and time-varying UEs' traffic densities, then the traffic densities will change the bias accordingly. The bias is uniquely determined from the traffic densitybias relationship.

To rebalance loads for LTE HetNet, the dynamic CRE is employed, whereby the low RSRP of an LPN is virtually biased to push cell edge HPN UEs to LPN cells actively. Therefore, a rule that dynamically associates the UEs to neighbouring LPN based on the load status of LTE HetNet is executed as per the designed algorithm in the following section.

CRE bias optimization implemented on LPN only, and the bias values dynamically change in proportion to the load status of each cell. Conventionally, UEs associate with the node that has the highest measured Reference Signal Received Power (RSRP) value. To rebalance loads, CRE is employed, whereby the low RSRP of a LPN is virtually biased to actively push cell edge HPN UEs to LPN cells.

\section{Algorithm design}

The algorithm is based on the mathematical model discussed in Equation (3) of the previous section and summarized by the Pseudo Code presented in Table 1. The algorithm comprises of two main procedures: the node side and the UE side procedures. On the node side, the node computes traffic density based on load, where each node has a unique traffic density used to calculate the bias. All the nodes perform the same procedure in a synchronized manner. On the UE side, for the UE to associate with a node, it measures pilot signals from nodes that are possible targets and reports accordingly.

The efficient load-balancing algorithm is executed iteratively in searching for a bias that maximizes fairness index value as compared with 0.8 of the maximum fairness index value of the benchmarked algorithm. For each iteration, traffic density and control information are exchanged amongst LPNs and HPNs. We use the Jain Fairness Index value as our metric for fairness in the algorithm. 
Table 1: Proposed algorithm

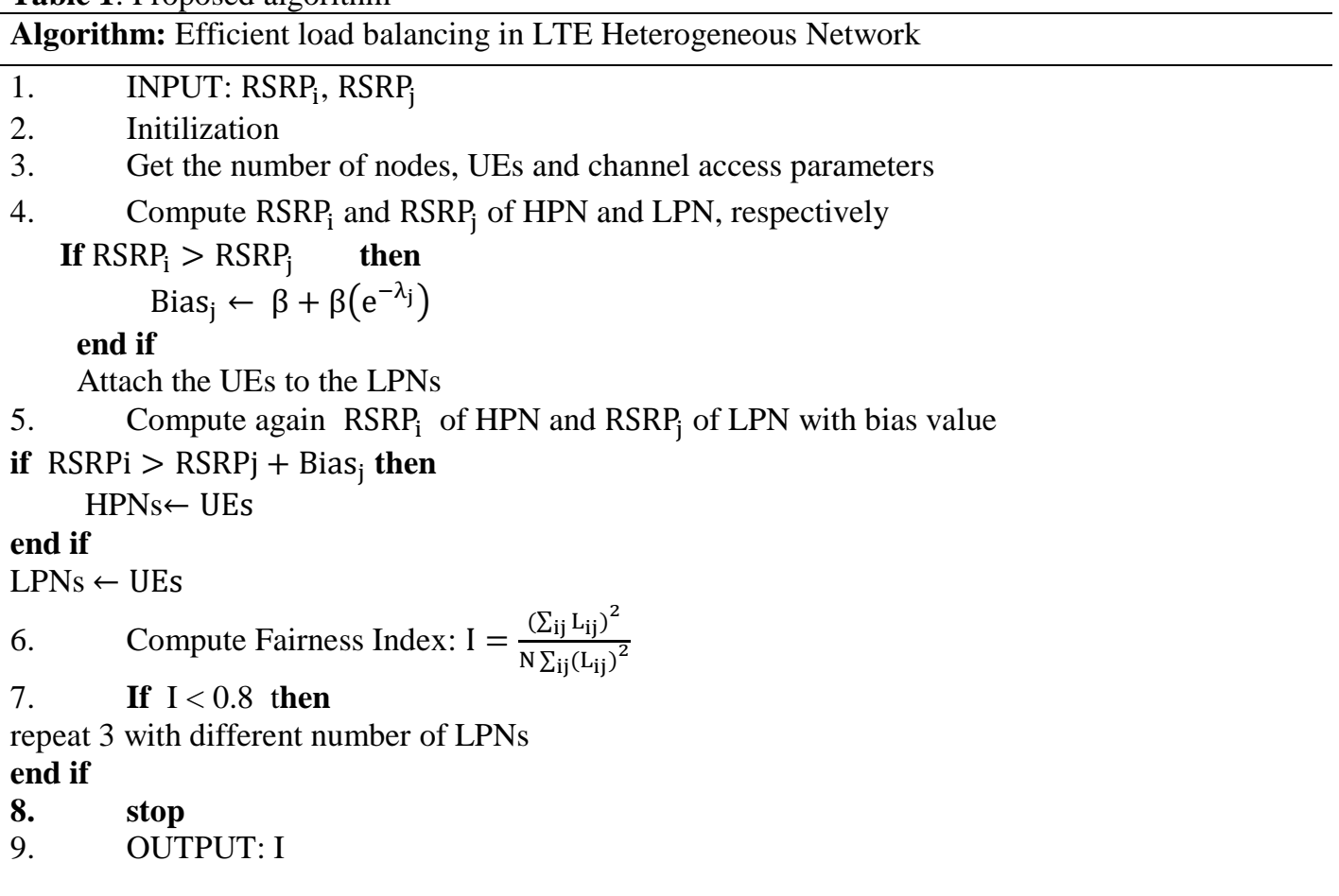

\section{Fairness in LTE Heterogeneous Network}

In LTE HetNet, fairness in resource allocation and scheduling is linked to sharing network resources evenly among the UEs. To evaluate fairness in our proposed algorithm, we used Jain's Fairness Index, $I$, defined in Equation (4) (Thienthong et al. 2019);

$$
I=\frac{\left(\sum_{i j} L_{i j}\right)^{2}}{N \sum_{i j}\left(L_{i j}\right)^{2}}
$$

where $N$ is the total number of samples taken and $L_{i j}$ signifies the load of HPN $i$ and LPN $j$. The values of $I$ range from $1 / N$ to 1 , which indicates the extent to which a system is fair. In our case, $1 / N$ represents an extreme case of an unbalanced network in which the load is heavily skewed towards one node, which takes the whole load. This is practically not possible since nodes are spatially distributed. On the other hand, when the fairness returns a value of one, it means the load is equally shared amongst the node. The performance of the algorithm, in terms of fairness, will, therefore, be determined by the load-balancing index.

\section{Results and Discussions}

Vienna LTE System Level simulator (Vienna-LTE-A-SLS-v1-9- Q1-2020) toolbox in MATLAB software was used to implement the developed load balance algorithm. A network consisting of 7 hexagonal HPN was considered, which has three sectors. Each of the sectors has four uniformly distributed LPNs, which are deployed as hotspots. Furthermore, 60 UEs were randomly placed within each sector where $3 / 4$ of the users were positioned around LPNs and the remaining were spread around HPN coverage areas. The main idea behind the described placement of users is to emulate a realistic distribution, as presented in Pham and Hwang (2017). Vienna LTE System Level simulator was used to collect the performed data by varying the number of LPNs. To include an urban HPN cell 
environment where UEs are located outdoors, the ITU-R urban Macro (UMa) channel model is chosen. Then for LPN cell environment, where signal propagation is from outdoors to indoors, and there is an expectation of concentrated UEs density, the ITU-R urban Micro (UMi) channel model is chosen. Table 2 summarizes the parameters used for simulation.

Table 2: Parameters used for simulation

\begin{tabular}{lll}
\hline Attribute & HPN & LPN \\
\hline Centre frequency & $2 \mathrm{GHz}$ & $2 \mathrm{GHz}$ \\
Network layout & $7 \mathrm{HPN}, 3$ sectors & $50 \mathrm{LPNs}$ \\
Path-loss model & ITU-R urban macro & ITU-R urban micro \\
Shadowing standard deviation & $4 \mathrm{~dB}(\mathrm{LoS}), 6 \mathrm{~dB}$ (nLoS) & $3 \mathrm{~dB}(\mathrm{LoS}), 4 \mathrm{~dB}(\mathrm{nLoS})$ \\
Bandwidth & $20 \mathrm{MHz}$ & $20 \mathrm{MHz}$ \\
Maximum transmit power & $46 \mathrm{dBm}$ & $30 \mathrm{dBm}$ \\
Number of UEs per sector & 60 & 60 \\
Traffic model & Full buffer & Full buffer \\
Inter site distance & $500 \mathrm{~m}$ & $100 \mathrm{~m}$ \\
\hline
\end{tabular}

The throughput, fairness and UEs offloading effect were used to quantify the performance. The proposed algorithm's performance was compared with the recent CRE based technique coupled with a particle swarm optimization (PSO) algorithm (Kuribayashi et al. 2020).

Figure 2 shows the variation of the throughput with the number of LPNs for both existing and proposed algorithms. It is observed that as the number of LPNs increases, throughput increases. This is possibly due to the fact that LTE HetNet throughput is highly affected by the traffic load congestion, as more LPNs tend to minimize traffic load congestion in LTE HetNet. From Figure 2, the average maximum achieved throughput of the existing and proposed algorithm are $10.56 \mathrm{Mbps}$ and $11.77 \mathrm{Mbps}$, respectively which is around $11 \%$ improvement.
The fairness was computed considering the entire nodes in a shared network in order to identify how the throughput is distributed in the network among various numbers of nodes. Figure 3 shows the variation of fairness with the number of LPNs for LTE HetNet for the existing and proposed algorithms. From the figure, it is observed that the fairness index value increases as the number of LPNs increases and the maximum fairness index of the proposed alogorithm improved by around $6 \%$ relative to the existing algorithm.

Figure 4 presents the average load distribution per UE in HPN as a function of the number of LPNs for the existing and proposed algorithms. It demonstrates the shift of HPN users to LPNs, whereby $39 \%$ of the UEs are overloaded HPN and $61 \%$ of UEs associated with underloaded LPN. 


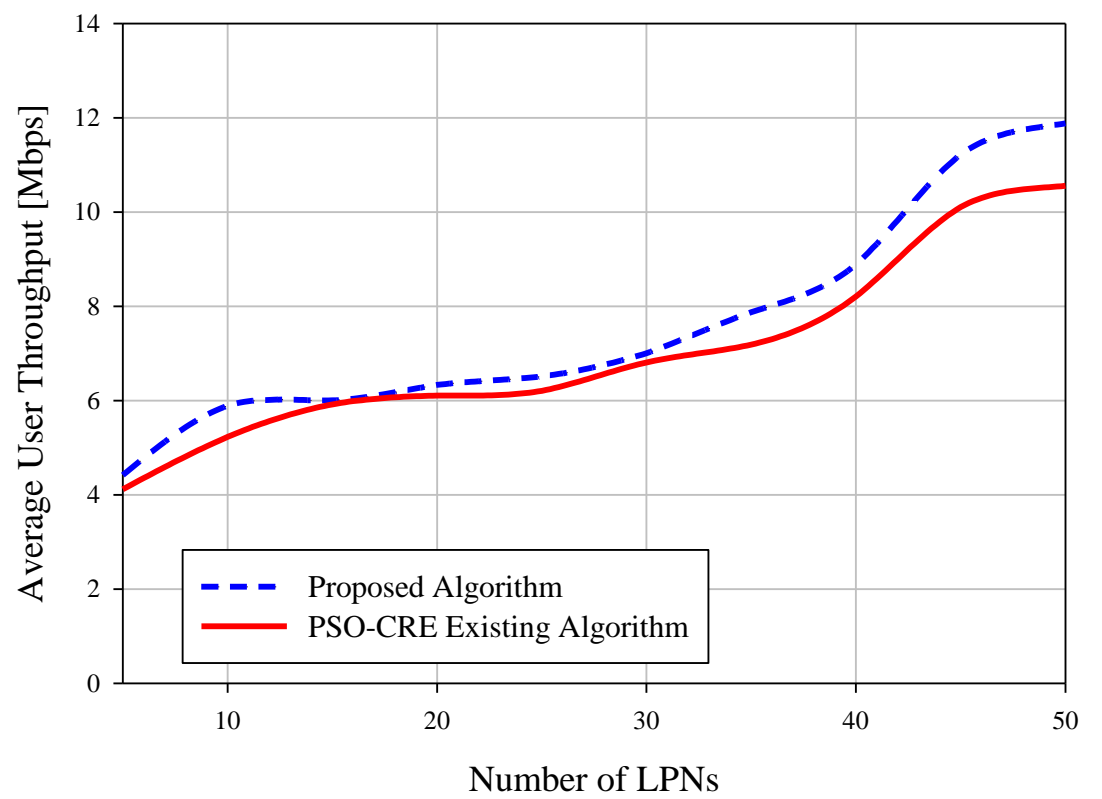

Figure 2: Variation of throughput with the number of LPNs for LTE HetNet.

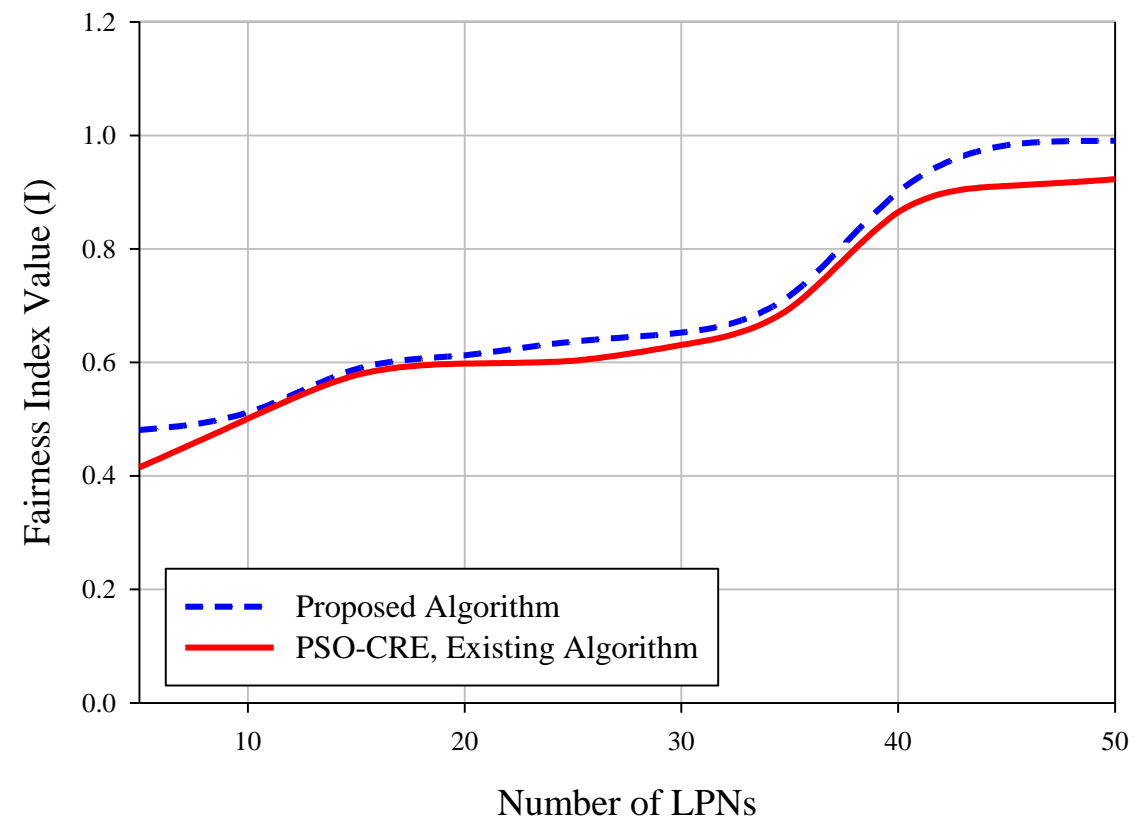

Figure 3: Variation of fairness with number of LPN for LTE HetNet. 


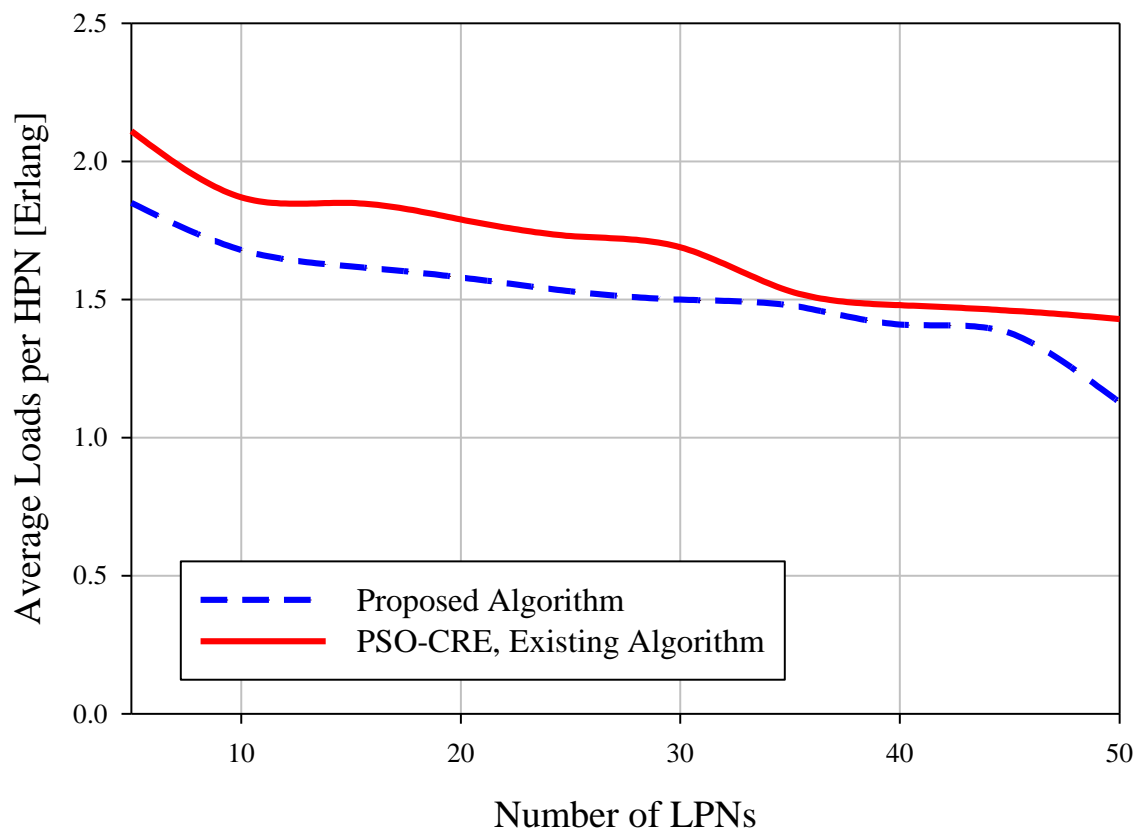

Figure 4: Load Distribution in HPN for LTE HetNet.

When the number of LPNs reaches 50, there is a significant improvement of around $30 \%$ on offloading effect relative to the existing algorithm. It is used to ascertain the responsiveness of the proposed load-balancing algorithm in HPN UEs offloading to LPNs. Table 3 summarizes the numerical superiority of the proposed algorithm in terms of Fairness Index Value, Average user throughput and UE offloading effects.

Table 3: Summary of performance improvement of the proposed algorithms

\begin{tabular}{llll}
\hline Performance metric & Existing algorithm & Proposed algorithm & Improvement (\%) \\
\hline Max Fairness Index Value & 0.92 & 0.98 & 6 \\
Max average user throughput & $10.56 \mathrm{Mbps}$ & $11.77 \mathrm{Mbps}$ & 11 \\
Min average loads per HPN & 1.43 Erlang & 1.13 Erlang & 21
\end{tabular}

\section{Conclusions}

This study introduced a mechanism to efficiently regulate traffic load balancing in LTE HetNet. The performance of the LTE HetNet depends on the load status between HPN and LPN. The traffic load should therefore, be distributed fairly between these nodes to relieve the congestion on a particular node and hence, improve QoS. The proposed algorithm regulates the load size by setting the mechanism to spy on the network load congestion between nodes and offloading as required. Simulation results show that there is a noticeable improvement in user throughput by $11 \%$ and the Fairness Index Value improvement of about $6 \%$ among the contending nodes compared to the latest existing algorithm. As an extension of this work, one can integrate CRE approach with enhanced Intercell Cell Interference 
Coordination (eICIC) techniques to reduce the severity of interference experienced by cell edge users when large CRE biases are assigned.

\section{References}

Abohamra YA, Solymani MR, and Shayan YR 2017 Optimum scheduling based on beamforming for the fifth generation of mobile communication systems. In $20178^{\text {th }}$ IEEE Annual Information Technology, Electronics and Mobile Communication Conference (IEMCON) 332-339. Vancouver.

Alam MJ, El-Saleh AA, Tan CK, Ku I, Lee YL, and Chuah TC 2018 Improved joint cell association and interference mitigation for lte-a heterogeneous networks. In ISTT 2018-2018 IEEE 4th International Symposium on Telecommunication Technologies 1-4. IEEE, Selangor.

Alhammadi A Roslee M, Alias MY, Shayea I, Alraih S and Mohamed KS 2020 Auto tuning self-optimization algorithm for mobility management in LTE-A and $5 \mathrm{G}$ HetNets. IEEE Access. 8: 294-304.

Bendaoud F, Abdennebi M and Didi F 2018 Network selection in wireless heterogeneous networks: A survey. $J$. Telecommun. Inf. Technol. 4: 64-74.

Bhuvaneswari PT V, Indu S, Shifana NL, Arjun D and Priyadharshini AS 2015 An analysis on cell range expansion in $4 \mathrm{G} \mathrm{LTE}$ networks. In $20153^{\text {rd }}$ International Conference on Signal Processing, Communication and Networking, ICSCN 2015 1-6. Chennai.

Dogra A, Jha RK, and Jain S 2020 A Survey on beyond $5 \mathrm{G}$ network with the advent of 6G : Architecture and Emerging Technologies. IEEE Access. 9: 6751267547.

Hammad K, Moubayed A, Primak SL and Shami A 2018 QoS-Aware energy and jitter-efficient downlink predictive scheduler for heterogeneous traffic LTE networks. IEEE Trans. Mobile Comput. 17(6): 1411-1428.
Hasiandra BM and Iskandar 2017 Planning and performance analysis of downlink interband carrier aggregation for LTE-Advanced 3GPP Released 13. Proceeding of 2016 10th International Conference on Telecommunication Systems Services and Applications, TSSA 2016: Special Issue in Radar Technology.

Huang W and Zhang H 2019 Coverage and throughput analysis for dual connectivity in ultra-dense heterogeneous networks. In IEEE International Conference on Communications 1-6. IEEE, Shanghai.

Kaur S and Sharma T 2018 Efficient load balancing using improved central load balancing technique. In $20182^{\text {nd }}$ International Conference on Inventive Systems and Control (ICISC) 1-5. IEEE, Coimbatore.

Klaine PV, Imran MA, Onireti O, and Souza RD 2017 A survey of machine learning techniques applied to self-organizing cellular networks. IEEE Communications Surveys and Tutorials 19(4): 2392-2431.

Kosmopoulos I, Skondras E, Michalas A, and Vergados DD 2020 An efficient mobility management scheme for $5 \mathrm{G}$ network architectures. In $20205^{\text {th }}$ South-East Europe Design Automation, Computer Engineering, Computer Networks and Social Media Conference (SEEDACECNSM) 1-6. Corfu.

Kuribayashi HP, De Souza MA, Gomes DDA, Silva KDC, Da Silva MS, Weyl Albuquerque Costa JC, and Lisboa Frances CR 2020 Particle swarm-based cell range expansion for heterogeneous mobile networks. IEEE Access. 8: 37021-37034.

Lai WK and Liu JK 2018 Cell Selection and resource allocation in LTE-advanced heterogeneous networks. IEEE Access. 6: 72978-72991.

Mannem K, Rao PN, and Reddy SCM 2020 Handover Decision in LTE \& LTE-A based hetnets using regression heuristics of quality metrics (RHQM) for optimal load balancing. In 2020 Fourth International Conference on Inventive Systems and 
Bakuza et al. - Efficient Load Balancing Algorithm in Long Term Evolution (LTE) Network ...

Control (ICISC) 732-739. IEEE, Coimbatore.

Netalkar PP, Maheshwari S, and Raychaudhuri D 2020 Evaluation of network assisted handoffs in heterogeneous networks. In $202029^{\text {th }}$ International Conference on Computer Communications and Networks (ICCCN) 1-9. Honolulu.

Ni S, Zhao J, Yang HH, Quek TQS, and Gong Y 2018 Small cell range expansion with interference mitigation for downlink massive MIMO HetNets. In 2018 IEEE Global Communications Conference, GLOBECOM 2018 - Proceedings 1-7. IEEE, Abu Dhabi.

Padaganur SK and Mallapur JD 2017 A neural network based resource allocation scheme for $4 \mathrm{G}$ LTE heterogeneous network. In $20172^{\text {nd }}$ International Conference for Convergence in Technology, I2CT 2017 250-254. Mumbai.

Pal P, Kumar A, Kaur T, Sahib F, Kumar S and Rastogi U 2020 Vertical handoff in heterogeneous mechanism for wireless lte network-an optimal approach. In 2020 International Conference on Emerging Trends in Communication, Control and Computing (ICONC3) 7-11. Lakshmangarh.

Pham QV and Hwang WJ 2017 Fairness-aware spectral and energy efficiency in spectrumsharing wireless networks. IEEE Trans. Vehicular Technol. 66(11): 10207-10219.

Ramaswamy V and Correia JT 2019 Enhancing service availability of LTE-in-a-box systems using 3GPP-compliant strategies. In Proceedings-IEEE Military Communications Conference MILCOM 512-517. IEEE, Los Angeles.

Thienthong P, Teerasuttakorn N, Nuanyai K, and Chantaraskul S 2019 Comparative study of scheduling algorithms and almost blank subframe for LTE HetNets. In iEECON 2019-7 ${ }^{\text {th }}$ International Electrical Engineering Congress, Proceedings 19-22. IEEE, Los Angeles. 